\title{
Power and Spreading Gain Allocation in CDMA Data Networks for Services with a Relative Priority
}

\author{
Kwang-Seop Jung \\ School of Electronic and Electrical Engineering \\ Kyungpook National University, Taegu, Korea
}

Sun-Mog Hong

School of Electronic and Electrical Engineering

Kyungpook National University, Taegu, Korea

\author{
Eun-Young Park \\ School of Electronic and Electrical Engineering \\ Kyungpook National University, Taegu, Korea
}

\begin{abstract}
In this paper, we consider algorithmic approaches for effectively providing data services for mobile users with different levels of priorities in a DS-CDMA system. The priority level of a user is specified by a factor that is a weighting on the instantaneous data throughput of the user. We define the weighted instantaneous aggregate data throughput (or the weighted channel efficiency) and use it to characterize the performance of the prioritized data service. Our prioritized data service is implemented so that the weighted instantaneous aggregate data throughput is maximized via efficient power and spreading gain allocation. We obtain properties of an optimal allocation that provides the maximum weighted instantaneous aggregate data throughput. Using these properties, we develop an efficient algorithm for computing the optimal power and spreading gain allocation. We also propose two suboptimal algorithms that are very fast and simple to implement.
\end{abstract}

Keywords: CDMA data networks, prioritized data services

\footnotetext{
The original version of this chapter was revised: The copyright line was incorrect. This has been corrected. The Erratum to this chapter is available at DOI: 10.1007/978-0-387-35618-1_37 


\section{Introduction}

As demands increase for data services over mobile wireless networks, the effective use of limited radio resource (channel bandwidth) becomes an imperative in wireless mobile data communications $[1,2]$. Often the non-real-time data services are less sensitive to delays compared to realtime services, and can be provided on a best-effort basis. There has been significant work on exploiting the delay tolerance of the data services to achieve high aggregate data throughput via an efficient allocation of the radio resource. Specifically, attempts have been made on power control and spreading gain allocation to maximize the aggregate non-real-time data throughput in a direct-sequence code-division multiple access (DSCDMA) system $[2,3,4]$.

In this paper, we consider algorithmic approaches for effectively providing data services for mobile users with different levels of priorities in a DS-CDMA system. The priority level of a user is specified by a factor that is a weighting on the instantaneous data throughput of the user. Firstly we define the weighted instantaneous aggregate data throughput (or the weighted channel efficiency) and use it to characterize the performance of the prioritized data service. Our prioritized data service is implemented so that the weighted instantaneous aggregate data throughput is maximized via efficient power and spreading gain allocation. This allows us to avoid dictating a strict priority service in which a lower-priority user is serviced only if services for all higher-priority users have been completed. Obviously this strict priority service can lead to a waste of the limited radio resource, since it cannot adapt effectively to time-varying channel quality due to fading, shadowing, and distance loss [5]. Secondly, we obtain properties of an optimal allocation that maximizes the weighted instantaneous aggregate data throughput. Using these properties, we develop an efficient algorithm for computing the optimal power and spreading gain allocation. We also propose two suboptimal algorithms that are very fast and simple to implement.

\section{System Model}

We consider the uplink in the DS-CDMA mobile packet data network that consists of $M$ mobile data users and a single base station. The mobile users transmit streams of data packets over a common radio channel using their respective terminal-specific spreading codes. Let us denote by $g_{i}\left(g_{i}>0\right)$ the instantaneous channel gain between user $i$ and the base station at an instant. Also, let us denote by $w_{i}\left(w_{i}>0\right)$ the weighting factor on the instantaneous throughput of the user $i$. The 
value of $w_{i}$ specifies the priority level of user $i$. The larger relative to other users' the value $w_{i}$ is, the higher the priority level of user $i$ is. The priority levels are discrete. Let us denote the number of priority levels by $L$ and the weighting factor of the level $l$ by $w^{l}$, respectively. Assume that $w^{1}>\cdots>w^{L}$. Obviously, $w_{i} \in\left\{w^{l}: l=1, \ldots, L\right\}, i=1, \ldots, M$.

We assume that without loss of generality $w_{1} g_{1}>\cdots>w_{M} g_{M}$. Each user can use the transmit power in an interval of zero to the maximum power $p_{\max }$. The bit-energy-to-interference-power-spectral-density $E_{b} / I_{o}$ of user $i$ is given by

$$
\gamma_{i}=\frac{p_{i} g_{i} N_{i}}{q+\alpha \sum_{j=1, j \neq i}^{M} p_{j} g_{j}}
$$

where $p_{i}$ and $N_{i}$ are, respectively, the power and spreading gain of user $i, q$ is the background interference and thermal noise power, and $\alpha$ is a constant depending on statistical characteristics between the spreading codes of users. The weighted instantaneous aggregate throughput (weighted channel efficiency) is defined by the weighted sum of each user's instantaneous throughput. That is,

$$
C(\mathbf{p}, \mathbf{N})=\sum_{i=1}^{M} \frac{\beta w_{i}}{N_{i}} f\left(\frac{p_{i} g_{i} N_{i}}{q+\alpha \sum_{j=1, j \neq i}^{M} p_{j} g_{j}}\right),
$$

where $\beta$ is the ratio of the channel code rate to the number of bits per packet. The function $f$ represents the probability of a successful packet transmission. We assume that $f$ is differentiable and non-decreasing in $E_{b} / I_{o}$. In this work, we assume that spreading gains can be real numbers for the simplicity of our analysis. Since the priority levels are specified by the weighting factors, the ratio of a pair of weighting factors becomes a quotient of relative prioritization between the pair of corresponding priority levels. Note that the weighted channel efficiency (2) with $w_{i}=1, i=1, \ldots, M$, becomes the instantaneous aggregate throughput in [4].

\section{Jointly Optimal Power and Spreading Gain}

In order to maximize the weighted channel efficiency in the DS-CDMA networks, it is essential to mitigate the impact due to the multiple access interference (MAI) and time-varying channel quality. Specifically we maximize the weighted channel efficiency $C(\mathbf{p}, \mathrm{N})$ by efficiently allocating the power and spreading gain. This results in an optimization problem to obtain the optimal combination of power and spreading gain 
allocation such that

$$
\max _{\mathbf{p}, \mathbf{N}} C(\mathbf{p}, \mathbf{N})
$$

subject to $0 \leq p_{i} \leq p_{\max }$ and $N_{i}>0$ for $i=1, \ldots M$. We can fortunately apply results in [4] to represent the optimum spreading gains of the optimization problem (3) as a function of the respective transmission power of $M$ users.

Proposition 1 [4]: The optimal spreading gain $N_{i}^{*}$ of user $i$ is

$$
N_{i}^{*}=\frac{\eta^{*}\left(q+\alpha \sum_{j=1, j \neq i}^{M} p_{j} g_{j}\right)}{p_{i} g_{i}}
$$

$i=1, \ldots M$, where

$$
\eta^{*}=\arg \max _{\eta \geq 1} \frac{1}{\eta} f(\eta)
$$

Substituting (4) into (2), we can see that the weighted channel efficiency depends upon $\mathbf{p}$ only. As a consequence, Proposition 1 allows us to reduce the optimization problem into a much simpler power control problem as follows. $\tilde{C}(\mathbf{p})$ denotes $C\left(\mathbf{p}, \mathbf{N}^{*}(\mathbf{p})\right)$.

$$
\max _{\mathbf{p}} \tilde{C}(\mathbf{p})
$$

subject to $0 \leq p_{i} \leq p_{\max }, i=1, \ldots M$.

We obtained properties of an optimal solution to this power allocation problem. Two most important properties (necessary conditions) of the optimal solution are summarized as follows. Due to the limited space, proofs are omitted.

Lemma 1: Suppose that $\mathbf{p}^{*}=\left(p_{1}^{*}, \ldots, p_{M}^{*}\right)$ solves the optimization problem (6). Then, $p_{i}^{*}=0$ or $p_{i}^{*}=p_{\max }$ for all $i \in\{1, \ldots, M\}$.

Lemma 2: Suppose that $\mathrm{p}^{*}=\left(p_{1}^{*}, \ldots, p_{M}^{*}\right)$ solves the optimization problem (6), and suppose that $p_{j}^{*}=p_{\max }$ for some $j \in\{1, \ldots, M\}$. Then, $p_{i}^{*}=p_{\max }$ for all $i \in\{1, \ldots, M\}$ such that both $w_{i} g_{i}>w_{j} g_{j}$ and $w_{i} \geq w_{j}$ are satisfied.

Lemma 1 reduces further the power control problem into a simple power assignment problem, which can be solved more efficiently. Lemma 2 helps to reduce significantly the number of candidate solutions, thereby the computational cost required to verify their optimality. The following two lemmas are also useful in implementing an efficient algorithm for solving the optimization problem. Let $g_{\max }=\max \left\{g_{i}: i=1, \ldots, M\right\}$ 
and $g_{\min }=\min \left\{g_{i}: i=1, \ldots, M\right\}$.

Lemma 3: If $\frac{p_{\max } g_{1}}{q} \geq \frac{w^{1} g_{\max }}{\alpha w_{1} g_{1}}, p_{1}^{*}=p_{\max }$ and $p_{i}^{*}=0, i=2,3, \ldots, M$.

Lemma 4: If $\frac{p_{\max } g_{\max }}{q} \leq \frac{1}{\alpha} \frac{w^{L}}{M w^{1}-(M-2) w^{L}}, p_{i}^{*}=p_{\max }$ for all $i \in\{1, \ldots, M\}$.

We use the results of Lemmas 3 and 4 in implementing power allocation algorithms for obtaining an optimal or suboptimal solution to the problem (6). The allocation algorithms are described in the following section.

Recall that the weighted channel efficiency (2) without prioritization becomes the instantaneous aggregate throughput in [4]. Accordingly, the instantaneous aggregate throughput maximization in [4] is a special case of the weighted channel maximization problem (6). It is easy to show that Lemmas 2-4 for no prioritization reduce to their corresponding results in [4], respectively.

\section{Power and Spreading Gain Allocation Algorithms}

Our algorithm OPT solves the power assignment problem (6) to obtain an optimal solution. It generates efficiently all the candidate solutions satisfying the necessary condition of Lemma 2. OPT then compares their respective weighted channel efficiencies to determine an optimal solution. We also propose two suboptimal algorithms $S A 1$ and $S A 2$. They are very fast and simple to implement. We denote by $I_{p}$ and $I_{p}^{c}$, respectively, the index sets of users with $p_{\max }$ assigned and with no power assigned. It is convenient to represent by $i_{l}^{\text {min }}$ the index of the user in $I_{p}^{c}$ with the highest channel gain in priority level $l$, i.e., $i_{l}^{\text {min }}=\min \left\{i \in I_{p}^{c}: w_{i}=w^{l}\right\}, l=1, \ldots, L$, if it exists. It is also convenient to represent by $i^{m i n}$ the index of the user in $I_{p}^{c}$ with the smallest index without concern about priority levels, i.e., $i^{m i n}=\min \left\{i \in I_{p}^{c}\right\}$. The algorithms begin with $I_{p}=\phi$ and return $I_{p}$ as a suboptimal solution.

\section{Suboptimal Algorithm 1 (SA1)}

Step 1: Set $I_{p}=\phi$ and $\tilde{C}_{\max }=0$.

Step 2: For $l=1, \ldots, L$, find, if it exists, $i_{l}^{\text {min }}$, assign $p_{\max }$ to users in $I_{p} \cup\left\{i_{l}^{\min }\right\}$ and compute its weighted channel efficiency $\tilde{C}_{l}$ for this assignment.

Step 3: Find $\bar{l}=\arg \max \left\{\tilde{C}_{l}: l=1, \ldots, L\right\}$.

Step 4: If $\tilde{C}_{\bar{l}}<\tilde{C}_{\max }$, stop and return $I_{p}$. Otherwise, update the index set $I_{p}$ by $I_{p} \cup\left\{i_{\bar{l}}^{\min }\right\}$. Update $I_{p}^{c}$ by the complement of $I_{p}$ and $\tilde{C}_{\text {max }}$ by 
$\tilde{C}_{\bar{l}}$, respectively.

Step 5: If the cardinality of $I_{p}$ is equal to $M$, stop and return $I_{p}$. Otherwise, go to Step 2.

Suboptimal Algorithm 2 (SA2)

Step 1: Set $I_{p}=\phi$ and $\tilde{C}_{\max }=0$.

Step 2: Find $i^{\text {min }}$. Assign $p_{\max }$ to users in $I_{p} \cup\left\{i^{\min }\right\}$ and compute its weighted channel efficiency $\tilde{C}_{0}$ for this assignment.

Step 3: If $\tilde{C}_{0}<\tilde{C}_{m a x}$, stop and return $I_{p}$. Otherwise, update the index set $I_{p}$ by $I_{p} \cup\left\{i^{m i n}\right\}$. Update $I_{p}^{c}$ by the complement of $I_{p}$ and $\tilde{C}_{\text {max }}$ by $\tilde{C}_{0}$, respectively.

Step 4: If the cardinality of $I_{p}$ is equal to $M$, stop and return $I_{p}$. Otherwise, go to Step 2.

$S A 1$ and $S A 2$ are not necessarily intended to generate a solution satisfying the necessary conditions, but they give a solution satisfying the conditions. In Step 2 of $S A 1$ and $S A 2$, we compute the weighted channel efficiency for a given power assignment $\mathbf{p}$ using the optimal spreading gain $\mathbf{N}^{*}(\mathbf{p})$ of (4) corresponding to this assignment. In fact, the spreading gain allocation of (4) is always assumed for a given optimal or suboptimal power allocation in this paper.

When $w^{l}$ are identical for all $l=1, \ldots, L, O P T, S A 1$, and $S A \mathscr{2}$ are basically identical and give an optimal solution. This is also true when $w^{l+1} / w^{l}, l=1, \ldots, L-1$, are sufficiently small. ${ }^{1}$ We implemented an algorithm $(M A X)$ for maximizing the unweighted instantaneous aggregate data throughput, and a strict priority service algorithm $(S T R)$. $S T R$ implements a strict priority service in which a lower-priority user is serviced only if services for all higher-priority users have been completed. Note that $M A X$ and $S T R$ solve the problem (6), respectively, for the above two special cases: $w^{l}, l=1, \ldots, L$, are identical and $w^{l+1} / w^{l}$, $l=1, \ldots, L-1$, are sufficiently small.

\section{Numerical Experiments}

Numerical experiments were performed in C-language on a 933-MHz Pentium III PC. First we compared the performances of our OPT, SA1, and $S A 2$ algorithms for $M=6$ and $M=12$ with $L=3$. The weighting factors of the three priority levels were set to $1.0,0.5$, and 0.25 , respectively. The number of users in each level is assumed two and four for $M=6$ and $M=12$, respectively. We obtained the computational time and weighted aggregate instantaneous throughput for each allocation 
Table 1. Performance Comparison of OPT, SA1, and SA2 Algorithms.

\begin{tabular}{lcccccc}
\hline & \multicolumn{3}{c}{$M=6$} & \multicolumn{3}{c}{$M=12$} \\
\hline & $O P T$ & $S A 1$ & $S A 2$ & $O P T$ & $S A 1$ & $S A 2$ \\
\hline Weighted channel efficiency & 1.52 & 1.52 & 1.52 & 2.63 & 2.63 & 2.63 \\
Computational time $(\mu \mathrm{s})$ & 6.31 & 1.31 & 0.16 & 35.1 & 1.48 & 0.16 \\
\hline
\end{tabular}

Table 2. Aggregate and Weighted Aggregate Data Throughput ${ }^{a}$ of $M A X, S A 2$, and $S T R$ Algorithms.

\begin{tabular}{lcccccc}
\hline & \multicolumn{3}{c}{$M=6$} & \multicolumn{3}{c}{$M=12$} \\
\hline & $M A X$ & $S A 2$ & $S T R$ & $M A X$ & $S A 2$ & $S T R$ \\
\hline Weighted Aggregate Throughput $^{\text {Aggregate Throughput }}{ }^{b}$ & 1.13 & 1.24 & 0.88 & 0.68 & 0.95 & 0.43 \\
\hline
\end{tabular}

${ }^{a}\left(\times 10^{-1}\right) .{ }^{b}$ packets/user/s.

update. For this comparison each user is assumed to have an infinite supply of packets. The distance model in $[2,6]$ with log-normal fading is assumed with the parameter set similar to the one given in [2]. We assumed $q=-97 \mathrm{~dB}, p_{\max }=100 \mathrm{~mW}$, and $\alpha=1.0$.

Table 1 indicates that $S A 2$ is 39.4 and 219.4 times faster than $O P T$, respectively, for $M=6$ and $M=12$. They also indicate that $S A 2$ is 8.19 and 9.25 times faster than $S A 1$, respectively, for $M=6$ and $M=12$. Clearly, the computational advantage of $S A 2$ is more significant as the number of users increases. Compared to $O P T$, the weighted aggregate instantaneous throughput was $99.5 \%$ and $99.2 \%$, respectively, for $S A 1$ and $S A 2$ for $M=6$. They were $99.3 \%$ and $99.2 \%$, respectively, for $S A 1$ and $S A 2$ for $M=12$. These results indicate that the computational advantage of $S A 2$ is significant and it generates a suboptimal allocation that gives the weighted aggregate instantaneous throughput very close to the theoretical upper limit that we can achieve.

We also implemented the strict priority service algorithm $(S T R)$ and an algorithm $(M A X)$ for maximizing the unweighted instantaneous aggregate data throughput. The weighting factors were set to 1.0 and 0.25 , respectively. Only one user was assumed to be serviced with level 1. The numerical results of our experiment are presented in Table 2. The ratios of the weighted channel efficiency were 0.71 and 0.91 for $(S T R) /(S A 2)$ and $(M A X) /(S A 2)$, respectively, for $M=6$. The ratios of the unweighted channel efficiency were 0.29 and 0.91 for $(S T R) /(M A X)$ 
and $(S A 2) /(M A X)$, respectively, for $M=6$. The ratios were $0.45,0.72$, and $0.19,0.93$ for $M=12$. These ratios show that $S A 2$ is superior to $S T R$ in terms of both the weighted and unweighted channel efficiency. They also show that $S A 2$ performs a trade-off between channel efficiency and strict prioritization. In fact, $S A 2$ implements a relative prioritization at the cost of a graceful loss of channel efficiency. This relative prioritization might be useful for providing a certain level of premium data services against transmission delays due to congestion while keeping channel efficiency reasonably maintained.

\section{Conclusion}

In this paper, we considered algorithmic approaches for effectively providing data services for mobile users with different levels of priorities in a DS-CDMA system. Our algorithms implement a trade-off between channel efficiency and strict prioritization.

\section{Acknowledgments}

This work was supported by Korea Ministry of Information \& Communication under University Research Program.

\section{Notes}

1. $\frac{w^{l+1}}{w^{l}} \leq \frac{\alpha M p_{\max } g_{\min }}{q+\alpha M p_{\max } g_{\max }}, l=1, \ldots, L-1$. We can choose $\frac{w^{l+1}}{w^{l}}$ so that this inequality is satisfied, since $g_{\max }, g_{\min }^{-1}$ and $q$ are finite in practice. If $q \ll \alpha M p_{\max } g_{\max }$, the inequality approximately reduces to $\frac{w^{l+1}}{w^{l}}<\frac{g_{\min }}{g_{\max }}$.

\section{References}

[1] Garg, V. K. and Wilkes J. E. (1996). Wireless and Personal Communications Systems. Upper Saddle River, NJ: Prentice-Hall.

[2] Oh, S.-J., T. L. Olsen, and Wasserman, K. M. (2000). "Distributed power control and spreading gain allocation in CDMA data networks," in Proc. IEEE Infocom'00, pp. 379-385, Tel Aviv, Israel.

[3] Oh, S.-J. and Wasserman, K. M. (1999). "Dynamic spreading gain control in multi-service CDMA networks," IEEE J. Select. Areas Commun., vol. 17, no.5, pp. 918-927.

[4] Oh, S.-J. and Wasserman, K. M. (1999). "Optimality of greedy power control in DS-CDMA mobile networks," in Proc. ACM/IEEE 5th Annual Int. Conf. Mobile Comput. and Network. (MobiCom'99), Seattle, WA.

[5] Guo, Y. and Chaskar, H. (2002). "Class-based quality of service over air interfaces in 4G mobile networks," IEEE Commun. Magazine, vol. 40, no. 3, pp. 132-137.

[6] Stuber, G. L. (1997). Principles of Mobile Communication. Boston, MA: Kluwer Academic Publishers. 